\title{
Geometric phase of a central spin coupled to an antiferromagnetic environment
}

\author{
Xiao-Zhong Yuan, ${ }^{1}$ Hsi-Sheng Goan, ${ }^{2,3, *}$ and Ka-Di Zhu ${ }^{1}$ \\ ${ }^{1}$ Department of Physics, Shanghai Jiao Tong University, Shanghai 200240, China \\ ${ }^{2}$ Department of Physics, and Center for Theoretical Sciences, \\ National Taiwan University, Taipei 10617, Taiwan \\ ${ }^{3}$ Center for Quantum Science and Engineering, National Taiwan University, Taipei 10617, Taiwan
}

\begin{abstract}
Using the spin-wave approximation, we study the geometric phase (GP) of a central spin (signal qubit) coupled to an antiferromagnetic (AF) environment under the application of an external global magnetic field. The external magnetic field affects the GP of the qubit directly and also indirectly through its effect on the AF environment. We find that when the applied magnetic field is increased to the critical magnetic field point, the AF environment undergoes a spin-flop transition, a firstorder phase transition, and at the same time the GP of the qubit changes abruptly to zero. This sensitive change of the GP of a signal qubit to the parameter change of a many-body environment near its critical point may serve as another efficient tool or witness to study the many-body phase transition. The influences of the AF environment temperature and crystal anisotropy field on the GP are also investigated.
\end{abstract}

PACS numbers: 03.65.Vf, 03.65.Yz, 75.30.Ds

The notion of adiabatic geometric phase (GP) of a quantum system first discussed in the pioneer work of Berry [1] was generalized and extended to nonadiabatic 2] and noncyclic [3] evolutions. It was also generalized to mixed states and nonunitary evolutions [4 [6]. Recently, the close relation between the GP's and quantum phase transition (QPT) in many-body systems was suggested and investigated 7]. In the QPT witness studies, an auxiliary spin or signal qubit is introduced to couple with a many-body system (environment) and the notion of Loschmidt echo (or equivalently the decoherence factor of the qubit) [8] or quantum state fidelity [9] is used to investigate the quantum criticality. The signature of QPT is the dramatic decay of the asymptotic value of the Loschmidt echo or the quantum state fidelity at the critical point. That is, the closer the environment to the QPT, the smaller the asymptotic value of the Loschmidt echo or the decoherence factor of the signal qubit. Recently, the GP of an auxiliary qubit induced by a onedimensional $X Y$ spin chain (an external environment) was calculated and the result was used to study the criticality of a special case of the transverse Ising model [10]. It was found that the induced GP in the qubit changes dramatically at the critical point [10].

Antiferromagnets subjected to an external magnetic field attracted considerable attention over the years [11]. One of the interesting phenomena in antiferromagnetic (AF) materials under an applied magnetic field is the magnetic-field-induced spin-flop transition. When the applied magnetic field is increased to the critical field point, the AF polarization flips into the direction perpendicular to the field. This is called the spin-flop transition, a first-order phase transition. The phenomena of the spin-flop transition were observed experimentally

*Electronic address: goan@phys.ntu.edu.tw
[12, 13]. It is thus particularly interesting to investigate how a globally applied external field influences the GP of a signal qubit that is coupled to an $\mathrm{AF}$ environment especially when the magnetic field strength is near the critical field of the spin-flop transition. It is known that the values of the critical magnetic field of the spin-flop transition can be obtained using the spin-wave theory. We thus apply the spin-wave approximation to deal with the AF environment in a globally applied magnetic field at low temperatures and low-energy excitations. The spin-wave approach allows us to go beyond the Markovian approximation and the weak-coupling limit in the usual treatments for system-coupled-to-environment models. The influence of the temperature and the crystal anisotropy field on the GP will also be investigated.

The total Hamiltonian of our model [14, 15] can be written as $H=H_{S}+H_{S B}+H_{B}$, where

$$
\begin{aligned}
H_{S}= & -g \mu_{B} B S_{0}^{z}, \\
H_{S B}= & -\frac{J_{0}}{\sqrt{N}} S_{0}^{z} \sum_{i}\left(S_{a, i}^{z}+S_{b, i}^{z}\right), \\
H_{B}= & J \sum_{i, \vec{\delta}} \mathbf{S}_{a, i} \cdot \mathbf{S}_{b, i+\vec{\delta}}+J \sum_{j, \vec{\delta}} \mathbf{S}_{b, j} \cdot \mathbf{S}_{a, j+\vec{\delta}} \\
& -g \mu_{B}\left(B+B_{A}\right) \sum_{i} S_{a, i}^{z}-g \mu_{B}\left(B-B_{A}\right) \sum_{j} S_{b, j}^{z},
\end{aligned}
$$

are the Hamiltonians of the signal qubit (central spin), the coupling, and the environment, respectively. Her $g$ is the gyromagnetic factor and $\mu_{B}$ is the Bohr magneton. For simplicity, a significant interaction between the central spin and the environment is assumed to be of the Ising type with $J_{0}$ being the coupling constant. $J$ is the exchange interaction and is positive for the AF environment. We assume that the spin structure of the environment may be divided into two interpenetrating sublattices $a$ and $b$ with the property that all nearest neighbors 
of an atom on $a$ lie on $b$ and vice versa. Each sublattice contains $N$ atoms with spin $S$, and $\mathbf{S}_{a, i}\left(\mathbf{S}_{b, j}\right)$ represents the spin operator of the $i$ th ( $j$ th) atom on sublattice $a$ (b). The indices $i$ and $j$ label the $N$ atoms, whereas the vectors $\vec{\delta}$ connect atom $i$ or $j$ with its nearest neighbors. $B$ represents a uniform external magnetic field applied in the $z$ direction. The anisotropy field $B_{A}$ is assumed to be positive, which approximates the effect of the crystal anisotropy energy, with the property of tending for positive magnetic moment $\mu_{B}$ to align the spins on sublattice $a$ in the positive $z$ direction and the spins on sublattice $b$ in the negative $z$ direction.

Using the Holstein-Primakoff transformation to map the spin operators of the $\mathrm{AF}$ environment onto bosonic operators, considering the situation that the environment is in the low-temperature and low-excitation limits, then transforming the resultant Hamiltonians to the momentum space, and finally using the Bogoliubov transformation, we obtain in the spin-wave approximation [15]

$$
\begin{aligned}
H_{S B} & =-\frac{J_{0}}{\sqrt{N}} S_{0}^{z} \sum_{\mathbf{k}}\left(\beta_{\mathbf{k}}^{\dagger} \beta_{\mathbf{k}}-\alpha_{\mathbf{k}}^{\dagger} \alpha_{\mathbf{k}}\right) \\
H_{B} & =\sum_{\mathbf{k}} \omega_{\mathbf{k}}^{(+)}\left(\alpha_{\mathbf{k}}^{\dagger} \alpha_{\mathbf{k}}+\frac{1}{2}\right)+\sum_{\mathbf{k}} \omega_{\mathbf{k}}^{(-)}\left(\beta_{\mathbf{k}}^{\dagger} \beta_{\mathbf{k}}+\frac{1}{2}(5)\right.
\end{aligned}
$$

where $\alpha_{\mathbf{k}}^{\dagger}\left(\alpha_{\mathbf{k}}\right)$ and $\beta_{\mathbf{k}}^{\dagger}\left(\beta_{\mathbf{k}}\right)$ are the creation (annihilation) operators of the two different magnons with wave vector $\mathbf{k}$ and frequency $\omega_{\mathbf{k}}^{(+)}\left(\omega_{\mathbf{k}}^{(-)}\right)$, respectively, and $\hbar=1$ in Eq. (5). For a cubic crystal system in the small $k$ approximation

$$
\omega_{\mathbf{k}}^{( \pm)}=2 M S J \sqrt{\left(1+\frac{g \mu_{B} B_{A}}{2 M S J}\right)^{2}+2 \frac{k^{2} l^{2}}{M}-1} \pm g \mu_{B} B,
$$

where $M$ is the number of nearest neighbors of an atom and $l$ is the side length of cubic primitive cell of the sublattice.

We assume that the initial density matrix of the total systems is separable (i.e., $\rho(0)=|\psi\rangle\langle\psi| \otimes \rho_{B}$, where the density matrix of the environment $\rho_{B}=e^{-H_{B} / T} / Z$, where $Z$ is the partition function and the Boltzmann constant has been set to one. The initial state of the central spin is described by $|\psi\rangle=\sin \left(\theta_{0} / 2\right)|e\rangle+\cos \left(\theta_{0} / 2\right)|g\rangle$, where $|e\rangle,|g\rangle$ denote the excited and ground states of the qubit and $\theta_{0}$ is the polar angle of the initial state in the Bloch sphere representation with $|g\rangle$ at the north pole. The reduced density matrix of the qubit in the thermodynamics limit (i.e., $N \rightarrow \infty$ ) can be obtained, following the calculation in Ref. [15], to be

$$
\rho(t)=\left(\begin{array}{cc}
\sin ^{2} \frac{\theta_{0}}{2} & \frac{1}{2} \sin \theta_{0} e^{-i g \mu_{B} B t-\left(t / \tau_{0}\right)^{2}} \\
\frac{1}{2} \sin \theta_{0} e^{i g \mu_{B} B t-\left(t / \tau_{0}\right)^{2}} & \cos ^{2} \frac{\theta_{0}}{2}
\end{array}\right)
$$

(7) wh really isolated from the AF environmont if the qubit (7) is really isolated from the AF environment, i.e., $J_{0}=0$, The decoherence time $\tau_{0}$ is given by [15] $\tau_{0}=$ then the decoherence time $\tau_{0} \rightarrow \infty$. In this case, we ob-

$\sqrt{2} \pi /\left[J_{0} \sqrt{\left(\eta^{+}+\eta^{-}\right)}\right]$, where

$$
\eta^{ \pm}=\frac{1}{2} \int_{0}^{\infty} \frac{e^{-\omega_{\mathbf{k}}^{( \pm)} / T}}{\left(1-e^{-\omega_{\mathbf{k}}^{( \pm)} / T}\right)^{2}} x^{2} d x
$$

with $x=k l$. The decoherence time depends on the coupling strength to the environment, on the structure parameters and temperature of the environment, and on the external magnetic field.

To calculate the GP for the qubit undergoing nonunitary evolution, we use the gauge invariant expression derived in [4]

$$
\Phi=\arg \left(\sum_{k} \sqrt{\varepsilon_{k}(0) \varepsilon_{k}(\tau)}\left\langle\phi_{k}(0) \mid \phi_{k}(\tau)\right\rangle e^{-\int_{0}^{\tau} d t\left\langle\phi_{k}|\partial / \partial t| \phi_{k}\right\rangle}\right),
$$

where $\tau$ denotes the total evolution time, and $\varepsilon_{k}(\tau)$ and $\left|\phi_{k}(\tau)\right\rangle$ are the eigenvalues and corresponding eigenvectors of the reduced density matrix $\rho(t)$. One may view the GP factor defined in Eq. (9) as a weighted sum over the phase factors pertaining to the eigenvectors of the reduced density matrix. The eigenvalues and eigenstates of $\rho(t)$ in Eq. (7) can be obtained as

$$
\begin{aligned}
\varepsilon_{ \pm}(t)= & \frac{1}{2} \pm \frac{1}{2} \sqrt{\cos ^{2} \theta_{0}+e^{-2 t^{2} / \tau_{0}^{2} \sin ^{2} \theta_{0}}}, \\
\left|\phi_{ \pm}(t)\right\rangle= & \frac{1}{\sqrt{\sin ^{2} \theta_{0} e^{-2 t^{2} / \tau_{0}^{2}}+4\left[\varepsilon_{ \pm}(t)-\sin ^{2} \frac{\theta_{0}}{2}\right]^{2}}} \\
& \times\left\{\sin \theta_{0} e^{-i g \mu_{B} B t-t^{2} / \tau_{0}^{2}}|e\rangle\right. \\
& \left.+2\left[\varepsilon_{ \pm}(t)-\sin ^{2} \frac{\theta_{0}}{2}\right]|g\rangle\right\} .
\end{aligned}
$$

Substituting Eqs. (10) and (11) into Eq. (9) and carrying out some simple manipulations, we obtain the GP acquired at the time $\tau=2 \pi /\left(g \mu_{B} B\right)$ of a cyclic period

$$
\Phi=\int_{0}^{\tau} d t \frac{g \mu_{B} B \sin ^{2} \theta_{0} e^{-2 t^{2} / \tau_{0}^{2}}}{\sin ^{2} \theta_{0} e^{-2 t^{2} / \tau_{0}^{2}}+4\left(\varepsilon_{+}-\sin ^{2} \frac{\theta_{0}}{2}\right)^{2}} .
$$

The AF environment causes decoherence and influences the GP of the signal qubit. Our results obtained using the spin-wave approximation are valid only for not too large temperatures, much smaller than the Néel temperature. According to neutron diffraction studies, the Néel temperature of antiferromagnet TbAuIn is $35 \mathrm{~K}$ [16]. Some antiferromagnets may have higher Néel temperatures. So in the following analysis, the environmental temperature is restricted below $T / g \mu_{B}=2.5$ Tesla (i.e., $T \approx 3.4 \mathrm{~K}$ ).

We, next, discuss the GP acquired at the time $\tau=$ $2 \pi /\left(g \mu_{B} B\right)$ after the qubit completes a cyclic evolution tain from Eq. (12) the well-known accumulated geometric 


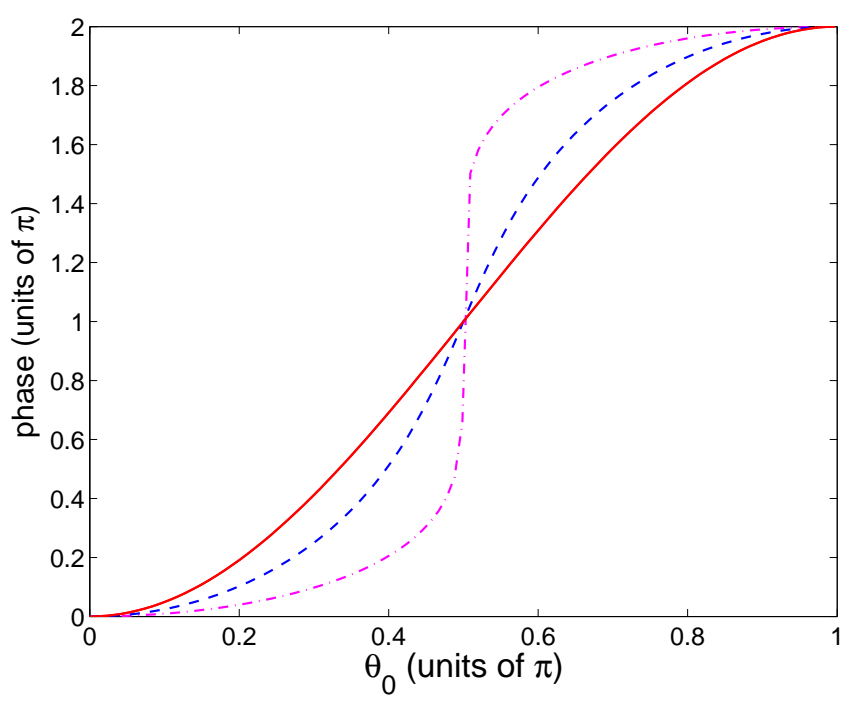

FIG. 1: (Color online) The GP of the signal qubit as a function of the polar angle $\theta_{0}$ for $J_{0}=0$ (solid curve), $J_{0}=2.5 \mathrm{~J}, T / g \mu_{B}=0.8$ Tesla (dashed curve), and $J_{0}=2.5 \mathrm{~J}$, $T / g \mu_{B}=1.2$ Tesla (dot-dashed curve). Other parameters are $M=6, M J / g \mu_{B}=40$ Tesla, $B_{A}=0.10$ Tesla, and $B=0.5$ Tesla

phase of a spin- $1 / 2$ particle processing around an external magnetic field in each cycle as: $\Phi=\pi\left(1-\cos \theta_{0}\right)$. If $\theta_{0}=\frac{\pi}{2}$, we have $\Phi=\pi$.

Figure 1 shows the GP of the qubit as a function of the initial polar angle $\theta_{0}$ for different temperatures. The external magnetic field is chosen as $B=0.5$ Tesla and other parameters are with $B_{A}=0.1$ Tesla and $M J / g \mu_{B}=40$ Tesla. The solid curve corresponds to the qubit isolated from the $\mathrm{AF}$ environment $\left(J_{0}=0\right)$ [i.e., $\left.\Phi=\pi\left(1-\cos \theta_{0}\right)\right]$. Comparing with the isolated case, we can see from Fig. [1 that the influence of the environment makes the GP curve deviate steeply from the isolated case in the region around $\theta_{0}=\pi / 2$. The deviation of the GP is positive for $\theta_{0}>\pi / 2$ and is negative for $\theta_{0}<\pi / 2$. Furthermore, the higher the temperature is, the steeper the curve is. For three special initial states with polar angles $\theta_{0}=0, \theta_{0}=\frac{\pi}{2}$, and $\theta_{0}=\pi$, the GP's are not affected by the $\mathrm{AF}$ environment.

In Fig. 2, we plot the GP as a function of external magnetic field $B$ for different temperatures with $\theta_{0}=1.3$. The solid curve corresponds to the isolated case $\left(J_{0}=0\right)$ [i.e., $\Phi=\pi(1-\cos 1.3)=0.73 \pi$ ], which is independent of $B$ for $B \neq 0$. It is obvious from Fig. 2 that the curve with higher temperature shows greater deviation from $\Phi=0.73 \pi$. For a given strength of the external magnetic field, the decoherence time $\tau_{0}$ of the off-diagonal elements of the reduced density matrix decreases with the increase of temperature. This reduces the GP obtained by the qubit [6]. The influence of the external magnetic field on the GP (12) can be categorized into two competing ways: one through the decoherence time $\tau_{0}$ and the other one through the quasiperiod $\tau=2 \pi /\left(g \mu_{B} B\right)$. If the external magnetic field increases, the decoherence time $\tau_{0}$ decreases [15]. Then it suggests that the influence of the environment on the GP increases with the increasing magnetic field. On the other hand, with the increase of the external magnetic field $B$, the quasiperiod $\tau$ decreases. It then takes less time for the environment to influence the GP in a quasicycle. In this sense, the influence of environment on the GP decreases with the increase of the external magnetic field. Hence, when the external magnetic field is very small, the decoherence time $\tau_{0}$ is finite [14, 15] but the qubit needs a rather long time $\tau \gg \tau_{0}$ to complete a quasicycle. Since the integrand with the factor $\exp \left(-2 t^{2} / \tau_{0}^{2}\right)$ in Eq. (12) becomes very small for time $t$ considerably larger than $\tau_{0}$, the integration over $t$ from $\tau_{0}$ to $\tau$ thus contributes very little to the whole GP of the signal qubit as compared to the isolated case. As a result, the dashed and dotdashed curves at very small $B$ show large deviation from the value $\Phi=0.73 \pi$ of the isolated case. One can furthermore obtain from Eq. (12) that the GP near $B=0$ behaves linearly in $B$ as $\lim _{B \rightarrow 0} \Phi=c_{1} B$, where $c_{1}$ is independent of $B$. As one increases the strength of the magnetic filed, the decoherence time decreases but the quasiperiod also decrease. Usually, the second effect is more important in typical values of the external magnetic field. Therefore, the low-temperature dashed curve approaches $\Phi=0.73 \pi$ with the increase of the external magnetic field. When the strength of the magnetic field is increased further toward the critical point where the spin-flop transition occurs, $B=B_{c} \approx 2.83$ Tesla for the parameters used in Figs. 2 and 3, the decoherence time

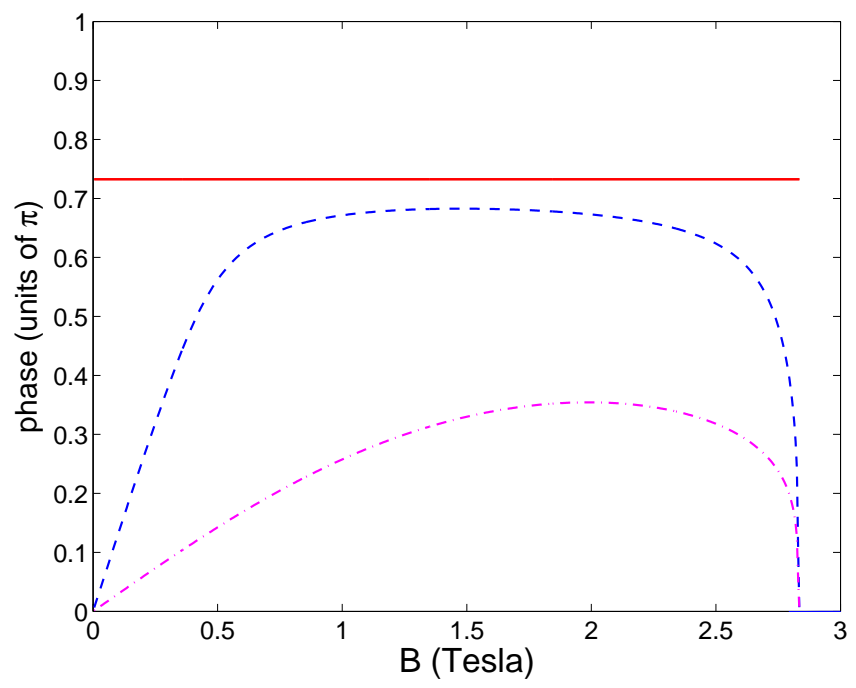

FIG. 2: (Color online) The GP of the signal qubit as a function of the external magnetic field $B$ for $J_{0}=0$ (solid curve), $J_{0}=2.5 \mathrm{~J}, \mathrm{~T} / g \mu_{B}=0.8 \mathrm{Tesla}$ (dashed curve), and $J_{0}=2.5 \mathrm{~J}$, $T / g \mu_{B}=1.5$ Tesla (dot-dashed curve). Other parameters are $\theta_{0}=1.3, M=6, M J / g \mu_{B}=40$ Tesla, and $B_{A}=0.10$ Tesla. 
approaches zero abruptly [15]. An analytical expression for the decoherence time near the critical field can be found in Eq. (39) of Ref. [15]. We note that this result using spin-wave theory is valid for $B<B_{c}$ (i.e., before the spin-flop transition). Thus when $B \rightarrow B_{c}$ from below, the environment exerts a great influence on the qubit so that its coherence is destroyed completely, but the quasiperiod is still finite. Such a great influence thus also appears on the GP and causes a sudden deviation from $\Phi=0.73 \pi$ for the dashed curves in Fig. 2 when the external magnetic field approaches $B_{c}=2.83$ Tesla. The drastic change in the induced GP of the qubit at the critical point is due to the sensitivity of the many-body (AF) environment to the parameter (the magnetic field) change near its critical point. Furthermore, from Eq. (39) of Ref. [15] and Eq. (12), one can obtain the GP near the critical field scaling as $\lim _{B \rightarrow B_{c}\left(B<B_{c}\right)} \Phi=c_{2}\left(B_{c}-B\right)^{1 / 4}$. Here $c_{2}$ is independent of the external magnetic field $B$. The linear behaviors of the GP near $B=0$ and the abrupt changes near $B=B_{c}$ can be clearly observed in Fig. 2

In Fig. 3. the GP of the qubit (central spin) is plotted as a function of $B$ and $T$ for the value of anisotropic field $B_{A}=0.10$ Tesla. Obviously, the curves in Fig. 2 are cross-section curves of the curved surface in Fig. 3 at different temperatures. As mentioned previously, one can observe from Fig. 3 that the GP decreases from the value $\Phi=0.73 \pi$ with the increase of the temperature. Besides, for $T / g \mu_{B}>0.5$ Tesla, it decreases quickly when $B$ approaches zero. As the external magnetic field approaches the critical point of $B_{c} \approx 2.83$ Tesla, the GP changes drastically to zero. Figure 4 is similar to Fig. 3] except that the anisotropy field is at $B_{A}=0.15$ Tesla. Then the spin-flop transition occurs at $B=B_{c} \approx 3.47$ Tesla. In such a case, the GP does not show abrupt change

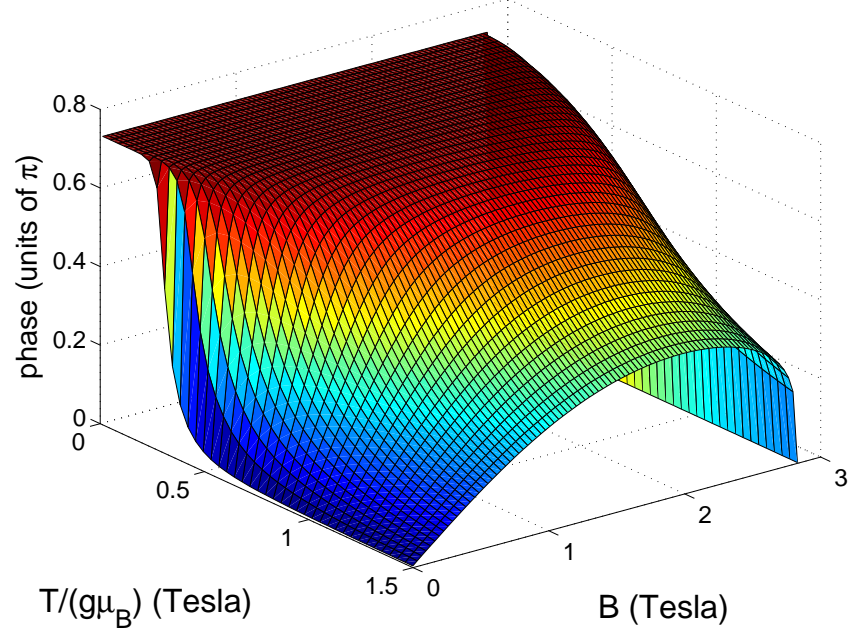

FIG. 3: (Color online) The GP of the signal qubit versus the external magnetic field $B$ and the environment temperature $T$. Other parameters are $\theta_{0}=1.3, M=6, M J / g \mu_{B}=$ 40 Tesla, $J_{0}=2.5 J$, and $B_{A}=0.10$ Tesla.

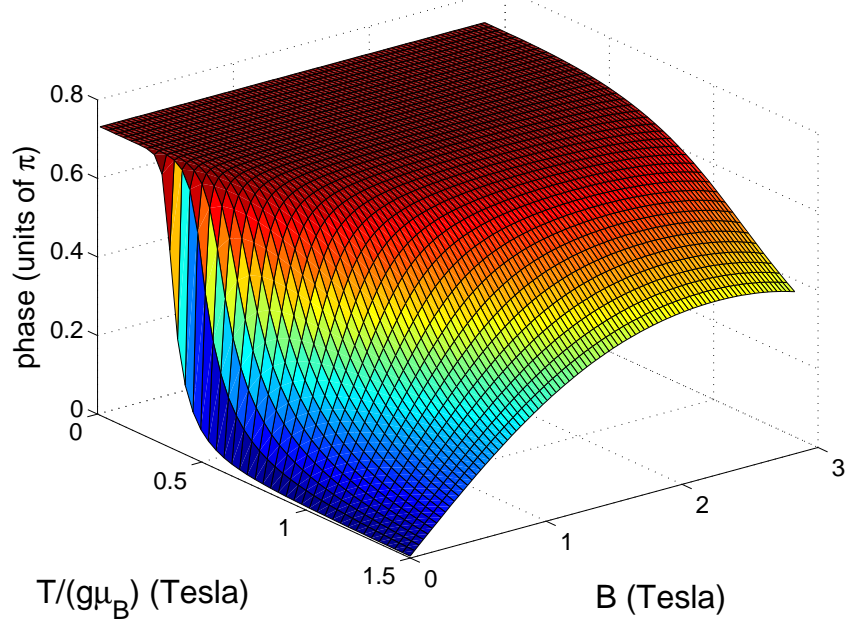

FIG. 4: (Color online) Same as Fig. 3, except $B_{A}=$ 0.15 Tesla.

even when $B$ approaches 3 Tesla. Comparing Fig. 3 with Fig. 4, we find that the large crystal anisotropy field suppresses the influence of the AF environment on the GP.

In conclusion, we investigated the influence of an $\mathrm{AF}$ environment on the GP of a signal qubit (central spin). Such an influence is enhanced by increasing the temperature or decreasing the crystal anisotropy field. The dependence of the GP on the external magnetic field $B$ involves two competing factors: quasiperiod $\tau$ and decoherence time $\tau_{0}$. A larger $B$ implies a smaller $\tau$ which indicates that the GP acquired at time $\tau$ by the qubit is less influenced by the environment in terms of the duration of interaction time with the environment. But the larger $B$ also implies smaller $\tau_{0}$ which indicates that the decays in the integrand of the GP (12) become greater. When $B$ approaches the region near the critical field of the spin-flop transition, $\tau_{0} \rightarrow 0$ but $\tau$ is finite. The qubit acquires no GP with $\tau_{0} \rightarrow 0$ and thus the GP changes abruptly at the critical field of the spin-flop transition. This sensitive change of the GP of a signal qubit to the parameter change of a many-body environment near its critical point may serve as, in addition to the Loschmidt echo and quantum state fidelity, another efficient tool or witness to study the phase transition.

H.S.G. acknowledges support from NSC under Grant No. 97-2112-M-002-012-MY3, from NTU under Grants Nos. 97R0066-65 and 97R0066-67, and from the NCTS focus group program. X.Z.Y. acknowledges support from NNSF of China under Grant No. 10874117. X.Z.Y. also thanks the conference support of ICTP (smr2046). 
[1] M. V. Berry, Proc. R. Soc. London, Ser. A 392, 45, (1984).

[2] Y. Aharonov et al., Phys. Rev. Lett. 58, 1593 (1987).

[3] J. Samuel et al., Phys. Rev. Lett. 60, 2339 (1988).

[4] D. M. Tong et al., Phys. Rev. Lett. 93, 080405, (2004).

[5] X. X. Yi et al., Phys. Rev. A 71, 044101 (2005); F. C. Lombardo and P. I. Villar, Phys. Rev. A 74, 042311, (2006).; X. Z Yuan and K. D. Zhu, Phys. Rev. B 74, 073309, (2006); X. L. Huang et al., Phys. Rev. A 78, 062114, (2008); K. Fujikawa and M. G. Hu, Phys. Rev. A 79, 052107, (2009).

[6] X. X. Yi et al., Phys. Rev. A 73, 052103, (2006);

[7] A. C. M. Carollo and J. K. Pachos, Phys. Rev. Lett. 95, 157203 (2005);S. L. Zhu, Phys. Rev. Lett. 96, 077206 (2006); A. Hamma, arXiv:quant-ph/0602091L. Campos Venuti and P. Zanardi, Phys. Rev. Lett. 99, 095701
(2007); Y. Q. Ma and S. Chen, Phys. Rev. A 79, 022116 (2009).

[8] H. T. Quan et al., Phys. Rev. Lett. 96, 140604 (2006); F. M. Cucchietti et al., Phys. Rev. A 75, 032337 (2007); D. Rossini et al., Phys. Rev. A 75, 032333 (2007).

[9] P. Zanardi and N. Paunković, Phys. Rev. E 74, 031123 (2006).P. Zanardi et al., Phys. Rev. A 75, 032109 (2007).

[10] X. X. Yi and W. Wang, Phys. Rev. A 75, 032103 (2007).

[11] A. Tanaka et al., Phys. Rev. B 79, 064412 (2009).

[12] G. P. Felcher et al., J. Appl. Phys. 50, 1837 (1979).

[13] U. Welp et al., Phys. Rev. Lett. 83, 4180 (1999).

[14] X. Z. Yuan et al., Europhys. Lett. 69, 868 (2005).

[15] X. Z. Yuan, H.-S. Goan, and K. D. Zhu, New J. Phys. 9, 219 (2007).

[16] L. Gondek et al., Solid State Commun. 136, 26 (2005). 